\title{
CHARACTERIZATION OF Sn FILMS ON SILICON BY SLOW POSITRON IMPLANTATION SPECTROSCOPY
}

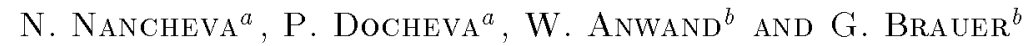 \\ ${ }^{a}$ Department of Physics, University of Rousse, 7017 Rousse, Bulgaria \\ ${ }^{b}$ Institut für Ionenstrahlphysik und Materialforschung, Forschungszentrum Rossendorf \\ Postfach 5101 19, 01314 Dresden, Germany
}

\begin{abstract}
Sn films grown on silicon substrate by d.c. magnetron sputtering have been investigated by slow positron implantation spectroscopy. As the substrate bias is one of the most important factors affecting the structure of a sputtered film, films grown at various substrate bias $(+80 \mathrm{~V}, 0 \mathrm{~V},-80 \mathrm{~V})$ are compared and their properties are discussed.
\end{abstract}

PACS numbers: $68.75 .+\mathrm{x}, 78.70$.

\section{Introduction}

The use of bias sputtering of films is a powerful method for controlling impurity concentration and adjusting composition [1-7]. Zhang et al. showed that the base bias voltage strongly influenced the film resistivity and transmittance during deposition and that a key way for obtaining a good film quality is controlled and adjusted precisely by a reactive gas partial pressure as well as a negative substrate bias $[1,2]$. By changing the bias potential, films with a wide range of conductivity [3] and structural and optical properties [7] can be deposited. Furthermore, there is a direct correlation of all the mechanical properties with bias voltage too [4]. The results in [5-7] show the optimum deposition conditions as indicated by an almost undamaged deposited layer [6], which are different from [7].

In this work, the role of biasing the substrate will be discussed in terms of the defect structure of sputter deposited Sn films on silicon substrates. This has been carried out by using slow positron implantation spectroscopy (SPIS), which is a suited method to characterize the structure of magnetron sputtered films [6]. 


\section{Experimental}

The Sn films, with the thickness $d$ of $1 \mu \mathrm{m}$, were deposited on high purity Si $p$-type plates $(\sim 0.5 \mathrm{~mm})$ by d.c. magnetron sputtering in argon atmosphere $\left(v_{\mathrm{d}}=100 \mathrm{~nm} / \mathrm{min}\right)$. The source material was $99.99 \%$ pure tin. During the deposition the current was kept constant $(I=0.2 \mathrm{~A})$. The sputtering system was arranged with a substrate carrier for application a negative and positive bias potential. The sputter target input power $(N=75 \mathrm{~W})$ and the total pressure $\left(P_{\text {total }}=8 \times 10^{-3}\right.$ mbar $)$ were kept constant with the bias stepped from $-80 \mathrm{~V}$ to $+80 \mathrm{~V}$

The SPIS was carried out at the slow positron beam "SPONSOR" at Rossendorf. The positrons are guided magnetically to the sample and can be accelerated in the energy range from $30 \mathrm{eV}$ to $35 \mathrm{keV}$ in steps of $100 \mathrm{eV}$. The energy resolution of the Ge detector at $511 \mathrm{keV}$ is $(1.09 \pm 0.01) \mathrm{keV} .7 \times 10^{5}$ events per spectrum at each incident positron energy have been accumulated.

\section{Results and discussion}

The SPIS data have been obtained for films deposited at bias potentials of $-80 \mathrm{~V}, 0 \mathrm{~V}$, and $+80 \mathrm{~V}$, respectively. One sample was measured from its back side in order to calculate exactly the substrate bulk characteristics of Si needed for the evaluation of the data measured on the deposited Sn layers. Using the software package VEPFIT [8] the Si bulk could be fitted to be $S_{\mathrm{b}}^{\mathrm{Si}}=0.4944$ and the positron

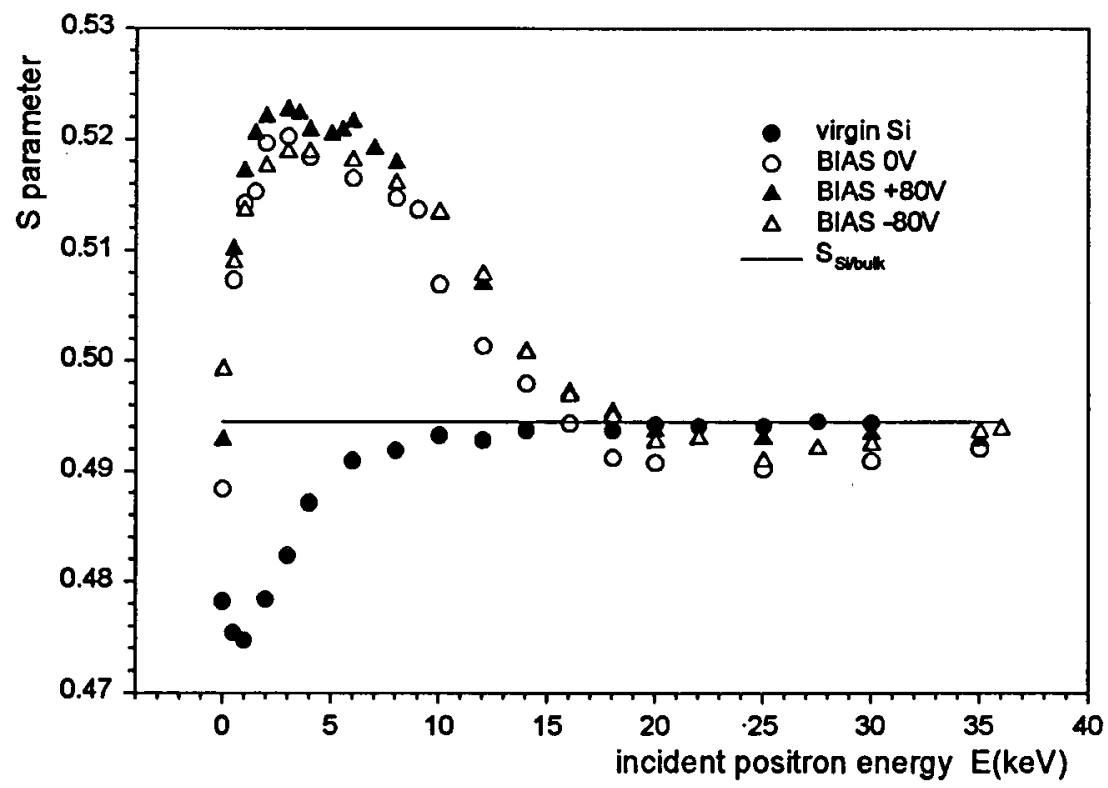

Fig. 1. S-parameter versus incident positron energy of magnetron sputtered Sn films on Si for different bias potentials $(+80 \mathrm{~V}, 0 \mathrm{~V},-80 \mathrm{~V})$. 


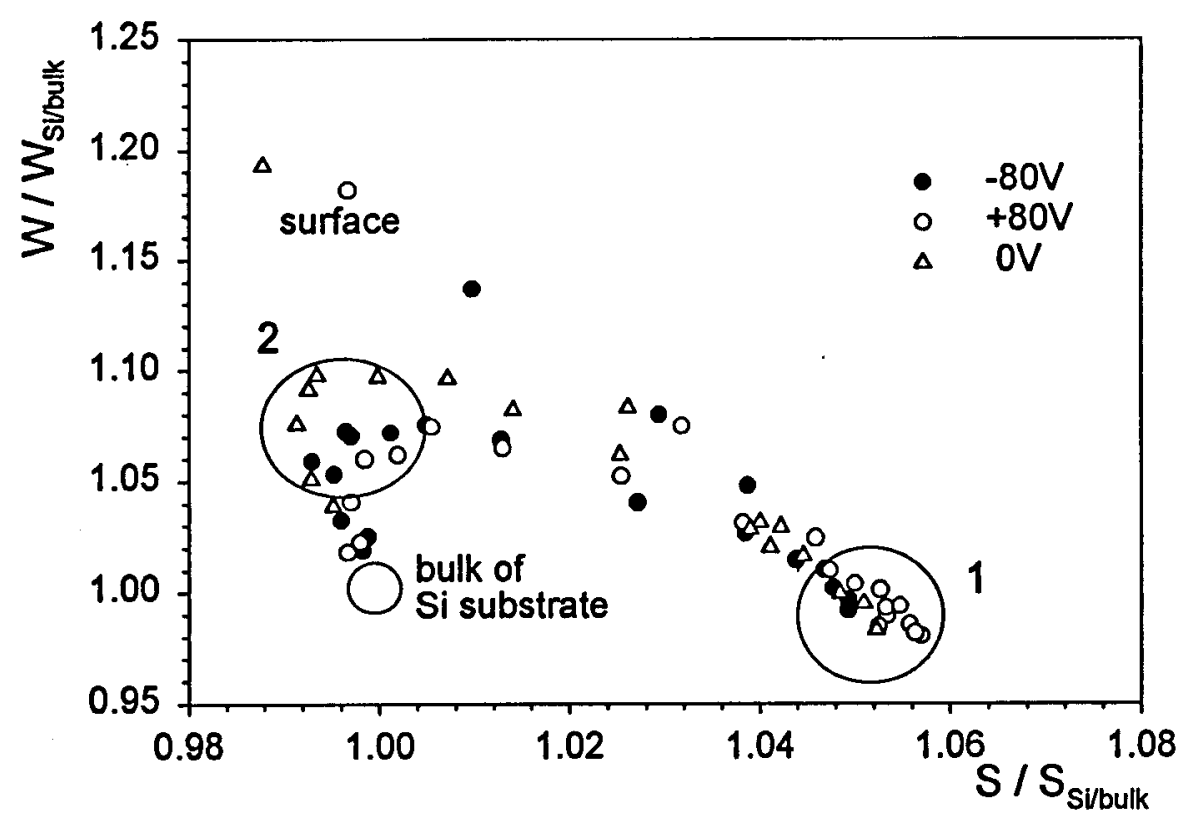

Fig. 2. $S$ versus $W$ plot of magnetron sputtered films with different bias potentials.

diffusion length $L_{+}^{\mathrm{Si}}=(213 \pm 20) \mathrm{nm}$. Figure 1 shows the $S$ parameter versus the incident positron energy $E$ of magnetron sputtered Sn films on Si substrate for different substrate bias potentials. At a small positron energy of $30 \mathrm{eV}$ the surface value of $S$ and $W$ depends on a lot of effects - an incomplete crystalline structure at the surface, oxidation, impurities. For this reason, it is impossible to explain, why $S(30 \mathrm{eV})<S(5 \mathrm{keV})$. One can only say, the positron annihilation at the surface takes place with low energy electrons.

In Fig. 2 the $S-W$ plot of the three magnetron sputtered samples is presented. The sputtering was carried out at different bias potentials. The $S-W$ plot gives a good overview about different annihilation states and at least three positron annihilation states are visible. Starting from the surface state a second state framed with circle 1 is reached characterized by a high $S$ parameter. The range framed with circle 2 is assigned to the deposited layer between the layer on the top and the Si substrate and characterized by a $S / S_{\mathrm{Si} / \text { bulk }}<1.0$. Due to the thick Sn layer we do not reach the bulk values of $S$ and $W$ of the Si substrate at the maximum of the incident positron energy as demonstrated by the empty circle. At positron energies of $35 \mathrm{keV}$ about $60-70 \%$ of the positrons annihilate in the Si substrate, the other part in the Sn layer. That means, $30-40 \%$ of the $S$ and $W$ parameter of the Sn layer contributes to the measured $S$ and $W$ parameter at $35 \mathrm{keV}$. For this reason, we do not measure the Si bulk values at $35 \mathrm{keV}$. From the $S-W$ plot no information about the depth depending defect structure can be extracted. For this reason, VEPFIT calculations have been done in order to class a typical value of the $S$ parameter to a depth in the sample. The results are summarized in the Table. 
TABLE

VEPFIT results.

\begin{tabular}{c|c|c|c}
\hline \hline bias [V] & $d[\mathrm{~nm}]$ & $S / S_{\text {bulk }}$ & $L_{+}[\mathrm{nm}]$ \\
\hline$-80 \mathrm{~V}$ & $0-269( \pm 6)$ & 1.0485 & $5 \pm 2$ \\
3-layers fit & $270-914( \pm 61)$ & 0.981 & $53 \pm 9$ \\
& deeper than & - & $213 \pm 20$ \\
& 914 nm Si substrate & & \\
\hline $0 \mathrm{~V}$ & $0-45( \pm 8)$ & 1.056 & $4 \pm 2$ \\
4-layers fit & $46-230( \pm 6)$ & 1.044 & $5 \pm 2$ \\
& $231-974( \pm 95)$ & 0.981 & $53 \pm 9$ \\
& deeper than & & $213 \pm 20$ \\
& 974 nm Si substrate & & \\
\hline 5-layers fit & $0-45$ & 1.061 & $4 \pm 2$ \\
& $46-79$ & 1.045 & $12 \pm 4$ \\
& $80-270$ & 1.068 & $65 \pm 10$ \\
& $270-780( \pm 76)$ & 0.986 & $58 \pm 13$ \\
& deeper than & & $213 \pm 20$ \\
& 780 nm Si substrate & &
\end{tabular}

For the fit of the magnetron sputtered Sn layer without bias potential a four-layer model has been used. The layer structure of the sample is already seen by naked eyes in Fig. 1. On the top of the sample we observe two layers with high $S$ parameters followed by a layer with an $S$ parameter lower than that of the Si substrate drawn in Fig. 1 as a line. That means, there are three layers characterized by different $S$ parameters on the top of the sample followed by the Si substrate. The characteristic depths of the layers in the Table are always related to zero at the surface. The layer thickness itself can be obtained by subtraction of the concerning layer boundaries.

The comparison of the biased and unbiased films results showed that the structure of the films depends on the bias voltage applied to substrate.

When the d.c. bias is applied to the substrate it accelerates different ions to a high energy so that they bombard the substrate, which not only changes the properties of deposited films, but also removes some of the deposited films. When the substrate has a negative bias potential it is bombarded mainly by $\mathrm{Ar}^{+}$ positive particles. Contrary to a positive bias voltage, the substrate is bombarded by negative particles.

Our data can be summarized as follows:

1. In the "back" experiment (Si substrate) $S_{\mathrm{b}}^{\mathrm{Si}}=0.4944, W_{\mathrm{b}}^{\mathrm{Si}}=0.1415$, positron diffusion lenght $L_{+}^{\mathrm{Si}}=213 \pm 20 \mathrm{~nm}$ and positron lifetime $\tau_{b}=218 \pm 3 \mathrm{ps}$. The value of lifetime is the same as in [9]. 
2. For all samples in the "face" case (Sn film) a few layers have been discovered. The total layer thickness sputtered to the substrates is about $800-1000 \mathrm{~nm}$. This points to a removal of some substrate and already deposited film during sputtering as the sputtered thickness should be $1 \mu \mathrm{m}$. Such effect has been described to exist in Refs. [1, 2, 6].

3. For the unbiased film $\left(U_{\mathrm{b}}=0 \mathrm{~V}\right)$ there are three layers on undamaged $\mathrm{Si}$ substrate. The surface layer is characterized by a high $S$ value (about $5.5 \%$ increase compared to the bulk) and a diffusion length $L_{+}=(4 \pm 2) \mathrm{nm}$. Another but less defected layer towards the substrate follows having about $4.4 \%$ increase in $S$ compared to the bulk and a diffusion length $L_{+}=$ $(5 \pm 2) \mathrm{nm}$. Open-volume defects should be smaller in size compared to the surface layer.

4. For the biased at $U_{\mathrm{b}}=-80 \mathrm{~V}$ film only two layers on undamaged Si substrate could be fitted. However, there is a hint for an additional layer between the first and the second layer, as seen from the graph in Fig. 1. The surface layer is characterized by a high $S$ value (about $4.8 \%$ increase compared to the bulk) and a diffusion length $L_{+}=(5 \pm 2) \mathrm{nm}$.

5. In the case of biased films at $U_{\mathrm{b}}=+80 \mathrm{~V}$ the deposited $\mathrm{Sn}$ film shows the most complicated structures. Three layers on the top of the substrate are characterized by high $S$ values (about $6.8 \%, 4.5 \%$, and $6.0 \%$ increase compared to the bulk, correspondingly) and different diffusion length (see the Table). The $S$ parameter of the layer, between $270 \mathrm{~nm}$ and $780 \mathrm{~nm}$ below the surface which consists of deposited Sn too, is a little bit higher than that measured for the other samples. This should be caused by vacancy type defects in this region.

All the above effects are related to the adatom mobility and the ion-solid interactions taking place close to the active growing surface during deposition. During bias sputtering initially there will always be a certain time interval before a monolayer is deposited onto the substrate surface. During this time interval the substrate will be sputter etched. In some bias intervals these etch depths become substantial before sputter etching is prevented by the deposited film layer. A bias applied to the substrate can attract ions in the plasma to bombard the growing film. This bombardment gives an additional energy to the particles condensed on substrate and peel off the atoms with a weak bonding from the film.

The results in [10] showed that when different bias voltages were applied to the substrate the net deposition rate decreased as compared in case without the substrate bias. Several effects may contribute to this: increasing in substrate etching by increasing ion current density to the substrate; substrate surface is modified by the ion bombardment; sticking coefficient of atoms to the substrate is decreased due to ion bombardment modification. The sputtering yield of a growing film changes somewhat from the point when the first atom adheres to the substrate to the point when the film almost completely covers the substrate surface. It is clear that the physical processes are too complicated in the magnetron plasma in order to estimate the influence of different electric and magnetic fields. 


\section{Conclusions}

As seen from our results the substrate bias plays an important role in the film growth. First investigations could demonstrate that the slow positron implantation spectroscopy can be a useful tool to clear up the depth depending structure of open-volume defects created by magnetron sputtering with different bias potentials.

\section{References}

[1] Huai-Wu Zhang, You-gang Zhang, Wu-yi Xu, Vacuum 45, 145 (1994).

[2] Huai-Wu Zhang, S.Q. Yang, Vacuum 46, 666 (1995).

[3] F. Arefi-Khonsari, F. Hellegouarc'h, J. Amouroux, J. Vac. Sci. Technol. A 16, 2240 (1998).

[4] P. Patsalas, C. Charitidis, S. Logothetidis, Surf. Coat. Technol. 125, 335 (2000).

[5] N. Nancheva, P. Docheva, M. Misheva, N. Djourelov, Bulgarian J. Phys. 25, 171 (1998).

[6] N. Nancheva, P. Docheva, W. Anwand, G. Brauer, P.G. Coleman, Acta Phys. Pol. A 95, 623 (1999).

[7] Z.W. Yang, S.H. Han, T.L. Yang, Lina Ye, D.H. Zhang, H.L. Ma, C.F. Cheng, Thin Solid Films 366, 4 (2000).

[8] A. van Veen, H. Schut, J. de Vries, R.A. Hakvoort, M.R. Ijpma, in: Positron Beams for Solids and Surfaces, Eds. P.J. Schultz, G.R. Massoumi, P.J. Simpson, American Institute of Physics, New York 1990, p. 171.

[9] W. Fuhs, U. Holzhauer, S. Mantl, F.W. Richter, R. Sturm, Phys. Status Solidi B 89, 69 (1978).

[10] S. Berg, C. Nender, B. Gelin, J. Vac. Sci. Technol. A 4, 448 (1986). 